\title{
Bacteriemias en pacientes oncológicos del Instituto del Cáncer SOLCA. Cuenca, 2011- 2016
}

\author{
Bacteriemia in cancer patients at the SOLCA Cancer Institute. \\ Cuenca, 2011-2016 \\ Mora Abad Johanna Magdalena', Procopio Adriana Nora², Hurtado \\ Bustamante Pablo Francisco', Alvarado Corral Raul Francisco ${ }^{1,3}$, \\ Martínez Reyes Fray Cleiton ${ }^{1,4}$
}

VOLUMEN 37 | No 1 | ABRIL 201

FECHA DE RECEPCIÓN: 22/01/2019 FECHA DE APROBACIÓN: 10/04/2019 FECHA PUBLICACIÓN: 30/04/2019

\section{SOLCA-Cuenca}

2. Hospital de Niños Dr. Ricardo Gutiérrez. Buenos Aires. Argentina

3. Hospital Santa Inés

4. Universidad del Azuay

\begin{tabular}{c|l}
$\begin{array}{c}\text { Artículo } \\
\text { original }\end{array}$ & $\begin{array}{l}\text { Original } \\
\text { Article }\end{array}$
\end{tabular}

Correspondencia:

cami_p2424ahotmail.com

Dirección:

Barbados 109

Código Postal:

010107

Teléfonos:

(07)2883124-0989561895

Cuenca - Ecuador

\section{RESUMEN}

Objetivo: caracterizar los episodios de bacteriemias, los microorganismos causantes y sus patrones de sensibilidad, en pacientes atendidos en el Instituto del Cáncer SOLCA - Cuenca, Ecuador.

Metodología: se utilizó un diseño descriptivo. El estudio se enfocó en todos los episodios de bacteriemias ocurridos en el periodo 2011-2016 verificados mediante hemocultivos. Las variables estudiadas fueron edad y sexo de los pacientes en los que se produjo la bacteriemia; tipo de tumor, microorganismo, tiempo de positivización y perfil de resistencia.

Resultados: se identificaron 318 episodios. El $66.8 \%$ de los microorganismos aislados fueron bacterias gramnegativas y el 33.2\% grampositivas; los más prevalentes fueron Escherichia coli 37.3\%, Staphylococcus aureus $17.9 \%$, Klebsiella.spp 9.3\%, Estafilococos coagulasa negativa $7.2 \%$ y Pseudomonas aeruginosa 5.1\%. En los cocos grampositivos, la meticilino resistencia fue de $40 \%$ en Staphylococcus aureus.y $67 \%$ en Staphylococcus coagulasa negativa; Escherichia coli y Klebsiella pneumoniae fueron resistentes a cefalosporinas de tercera generación en un $29 \%$ y $47 \%$, respectivamente, compatibles con el fenotipo de beta-lactamasa de espectro extendido; la resistencia a quinolonas fue $35 \%$ y $50 \%$ respectivamente.

Conclusiones: las bacterias gramnegativas fueron los microorganismos más prevalentes en este estudio, principalmente enterobacterias, con una importante resistencia a los antibióticos ensayados.

Palabras claves: bacteriemia, instituciones oncológicas, pacientes internos, cultivo de sangre, neoplasias, farmacorresistencia, microbiana. 


\section{ABSTRACT}

Objective: to characterize the episodes of bacteremia, the causative microorganisms and their patterns of sensitivity in patients treated at the Cancer Institute SOLCA - Cuenca, Ecuador.

Methodology: a descriptive design was applied. The study focused on all episodes of bacteremia occurred in the period 2011-2016, they were verified by blood cultures. The variables studied were age and sex of the patients in whom the bacteremia occurred; type of tumor, microorganism, time of positivization and resistance profile.

Results: a total of 318 episodes were identified. The $66.8 \%$ of the isolated microorganisms were gram-negative bacteria and $33.2 \%$ gram-positive; the most prevalent were Escherichia coli $37.3 \%$, Staphylococcus aureus $17.9 \%$, Klebsiella.spp $9.3 \%$, Staphylococcus coagulase negative $7.2 \%$ and Pseudomonasaeruginosa $5.1 \%$. In grampositive cocci, methicillin resistance was $40 \%$ in Staphylococcus.aureus and $67 \%$ in coagulasenegative Staphylococcus; Escherichia.coli and Klebsiella pneumoniae were resistant to thirdgeneration cephalosporins by $29 \%$ and $47 \%$ respectively, compatible with the extendedspectrum beta-lactamase phenotype; the resistance to quinolones was $35 \%$ and $50 \%$ respectively.

Conclusions: the gram-negative bacteria were the most prevalent microorganisms in this study, mainly the enterobacteria, with an important resistance to the antibiotics tested.

Keywords: bacteremia, cancer care facilities, inpatients, blood culture, neoplasms, drug resistance, microbial.

\section{Introducción}

La bacteriemia se define como la presencia de bacterias en la sangre; indica falla del sistema inmune; están reconocidas como una de las mayores causas de morbilidad y mortalidad a nivel mundial. En pacientes oncológicos son comunes y severas, principalmente en los que reciben quimioterapia por la neutropenia que provoca $y$, concomitantemente, son sometidos a procedimientos invasivos. El hemocultivo representa una parte fundamental para la identificación etiológica y un adecuado manejo terapéutico [1-4].
La incidencia en pacientes oncológicos depende del tipo de cáncer y alcanzan hasta el $15 \%$ por año; la mortalidad varía de acuerdo a lo señalado y al tipo de infección, llegando a ser mayor al $50 \%$ cuando las bacteriemias son por microorganismos resistentes, en los pacientes neutropéni$\cos [4]$.

El microorganismo aislado se relaciona con el foco; el tracto urinario, las vías respiratorias inferiores, el tracto gastrointestinal, las infecciones de piel y partes blandas son las localizaciones más comunes. Las infecciones urinarias y del tracto respiratorio inferior asocian a bacteriemia de la comunidad; las debidas a cuidados de la salud son, en primer lugar, las infecciones relacionadas con catéteres [5]. Aproximadamente del $25 \%$ al $30 \%$ cursan con foco desconocido; esto dificulta la identificación del lugar de origen y el microorganismo causante, por ende, la terapia antibiótica [6,7].

La expectativa de vida en los pacientes oncológicos ha mejorado de manera importante en los últimos tiempos; sin embargo la respuesta de los pacientes no siempre es satisfactoria y con cierta regularidad se puede producir inmunosupresión, incrementándose el riesgo de infecciones que pueden llevar incluso a la muerte [8].

El $80 \%$ de las bacteriemias en pacientes oncológicos en los años 70 del siglo pasado se debieron principalmente a E.coli, Klebsiella spp. y P.aeruginosa. Hace aproximadamente 20 años hubo un cambio en estos patrones: las bacterias grampositivas tomaron predominio siendo las más aisladas Estafilococo Coagulasa Negativo (ECN), S. aureus, Estreptococos del grupo viridans y Enterococos spp. $[4,5,9,10]$. No se conoce la causa de este cambio, pero se cree que el uso del catéter de larga permanencia y el contacto con superficies mucosas favorecería la entrada de ECN; para los estreptococos del grupo viridans se han evaluado posibles factores como la neutropenia, la mucositis oral, la administración de quimioterapia, la profilaxis con fluoroquinolonas $o$ cotrimoxazol, o bien, el tratamiento de la gastritis con antagonistas para el receptor de hidrógeno $[4,11,12]$

Diversos factores se relacionan con una evolución clínica adversa de las bacteriemias, algunos no modificables como la patología del huésped, 
enfermedades subyacentes, el origen de la infección o los microorganismos implicados; y otros modificables: la administración temprana de antibióticos apropiados, el tratamiento del foco de origen, o el soporte hemodinámico entre otros $[1,12,13]$.

\section{MATERIALES Y MÉTODOS}

El presente estudio es descriptivo; se lo efectuó con los episodios de bacteriemias de los pacientes ingresados en el Instituto del Cáncer SOLCA - Cuenca del periodo 2011-2016. Los criterios de inclusión fueron todos los episodios de bacteriemia verdadera verificados a través hemocultivos. Se consideró episodio de bacteriemia al primer aislamiento significativo de un hemocultivo, y todos los adicionales dentro de las 48 horas del primero, a menos que el foco siga siendo el mismo. Se consideró hemocultivo positivo al aislamiento en sangre periférica de un microorganismo reconocido usualmente como patógeno o que cumplía con los criterios generales de jerarquización de microorganismos en pacientes con clínica compatible; en el caso de la flora cutánea, al menos dos hemocultivos positivos con un mismo microorganismo (ECN, micrococos, corinebacterias, Bacillusspp, Propionibacterium acnes) con idéntica especie y sensibilidad antibiótica. Se registró como duplicación, el aislamiento del mismo agente bacteriano con la misma sensibilidad en el mismo paciente en forma consecutiva; en estos casos se consignó sólo una vez el microorganismo aislado, asumiendo que todos correspondían al mismo proceso febril. Se asumió como un nuevo episodio de bacteriemia cuando el hemocultivo de un mismo paciente se presentó positivo por otro microorganismo, posterior a las 48 horas del inicial.

La extracción y procesamiento de la muestra de sangre, se ejecutaron de acuerdo a los protocolos correspondientes. La identificación de los microorganismos se realizó con distintas técnicas, según disponibilidad: pruebas bioquímicas convencionales, kit RapIDOneSystem, RapID NF Plus System (remel, USA) para enterobacterias y BNF respectivamente, EstreptococcalGrouping Kit (OXOID, UnitedKingdom). La determinación de la susceptibilidad a los antimicrobianos fue hecha por el método de difusión con discos en agar Kirby Bauer, tomando siempre como referencia los puntos de corte establecidos por las recomen- daciones del Instituto de estándares del laboratorio clínico CLSI.

Se identificó también, la edad y sexo de los pacientes en quienes se produjeron los episodios de bacteriemia.

Dado que el estudio consistió en una revisión documental, las historias clínicas fueron tratadas de acuerdo con los principios de confidencialidad sólo para fines investigativos, sin comprometer la identidad de los pacientes. Se solicitó la autorización correspondiente siguiendo la normativa institucional.

Los datos recolectados fueron procesados en el programa estadístico SPSS V20 portable y el software WHONET 5.6 para los datos de susceptibilidad a los antimicrobianos. Se utilizó estadística descriptiva de acuerdo con las variables registradas.

\section{RESULTADOS}

Durante el período estudiado se documentaron 318 episodios de bacteriemias en 268 pacientes, $140(52 \%)$ correspondieron al sexo masculino y $128(48 \%)$ al femenino. La mediana de la edad en los primeros fue de 27 años, y en las mujeres de 48. De las bacteriemias, $4(1.3 \%)$ casos resultaron polimicrobianos con dos microorganismos: $P$. aeruginosa y E. coli, S. aureus y E. coli, K.pneumoniae y A. hydrophila, E. coli y K. oxytoca; se aislaron un total de 322 microorganismos.

Porcentualmente, los episodios de bacteriemia, de acuerdo al tipo de tumor, $57.5 \%$ correspondieron a tumores hematológicos, $30.3 \%$ a sólidos y $12.3 \%$ a linfomas.

Sobre el tipo de agente causal de las bacteriemias, los resultados mostraron lo siguiente: 


\section{Tabla No 1}

Distribución de los agentes etiológicos de las bacteriemias en pacientes oncológicos del Instituto del Cáncer SOLCA. Cuenca, 2011 - 2016

\begin{tabular}{|c|c|c|c|}
\hline \multicolumn{2}{|l|}{ Microorganismos } & \multirow{2}{*}{$\begin{array}{c}\mathrm{n} \\
125\end{array}$} & \multirow{2}{*}{$\begin{array}{c}\% \\
37.3\end{array}$} \\
\hline Bacterias Gramnegativas & Escherichiacoli & & \\
\hline & Klebsiellaspp & 31 & 9.3 \\
\hline & Pseudomonaaeruginosa & 17 & 5.1 \\
\hline & Burkholderiacepacia & 9 & 2.7 \\
\hline & Salmonellaspp & 9 & 2.7 \\
\hline & Enterobactercloacae & 7 & 2.0 \\
\hline & Morganellamorganii & 3 & 0.9 \\
\hline & Acinetobacterspp & 3 & 0.9 \\
\hline & Citrobacterfreundii & 2 & 0.6 \\
\hline & Aeromonashydrophila & 2 & 0.6 \\
\hline & Proteusmirabilis & 2 & 0.6 \\
\hline & Serratiamarcescens & 1 & 0.3 \\
\hline & Achromobacterxylosoxidans ss. xylosoxidans & 1 & 0.3 \\
\hline & Moraxellacatarrhalis & 1 & 0.3 \\
\hline & Stenotrophomonasmaltophilia & 1 & 0.3 \\
\hline & Pseudomonastutzeri & 1 & 0.3 \\
\hline \multirow[t]{9}{*}{ Bacterias Grampositivas } & Staphylococcusaureus & 60 & 17.9 \\
\hline & Staphylococcuscoagulasa negativa & 24 & 7.2 \\
\hline & Streptococcusbetahemolíticogrupo C & 6 & 1.8 \\
\hline & Streptococcuspneumoniae & 6 & 1.8 \\
\hline & Enterococcusspp & 6 & 1.8 \\
\hline & Listeria monocytogenes & 2 & 0.6 \\
\hline & Streptococcusviridans & 2 & 0.6 \\
\hline & Streptococcuspyogenes & 1 & 0.3 \\
\hline & Total & 322 & 100.0 \\
\hline
\end{tabular}

Elaborado por: los autores. Fuente: base de datos.

De la tabla $N^{0} 1$ se desprende que de los 322 microorganismos, 215 (66.8\%) fueron bacterias gramnegativas y 107 (33.2\%) grampositivas. 
Respecto al tiempo de positivización de los hemocultivos según el microorganismo aislado, se observó lo siguiente:

\section{Tabla No 2}

Microorganismos aislados en episodios de bacteriemia y tiempo de positivización en los hemocultivos del Instituto del Cáncer SOLCA. Cuenca, 2011 - 2016

\begin{tabular}{llcccc}
\hline Bacterias & & $\mathrm{n}$ & Mediana & \multicolumn{2}{c}{ Rango } \\
\hline Gramnegativas & Escherichiacoli & 125 & 12.0 & 6.0 & 34.0 \\
\hline & Klebsiellaspp & 31 & 12.0 & 8.0 & 16.0 \\
& Pseudomonaaeruginosa & 17 & 15.0 & 9.0 & 24.0 \\
& Burkholderiacepacia & 9 & 22.0 & 12.0 & 42.0 \\
& Salmonella spp & 9 & 14.0 & 10.0 & 16.0 \\
& Enterobactercloacae & 7 & 12.0 & 7.0 & 14.0 \\
& Acinetobacterspp & 3 & 12.0 & 11.0 & 20.0 \\
& Morganellamorganii & 3 & 15.0 & 12.0 & 19.0 \\
\hline Grampositivas & Staphylococcusaureus & 60 & 12.0 & 6.0 & 42.0 \\
& Staphylococcuscoagulasa negativa & 24 & 12.5 & 9.0 & 32.0 \\
& Streptococcusbetahemolítico grupo C & 6 & 12.0 & 10.0 & 18.0 \\
& Streptococcuspneumoniae & 6 & 12.0 & 10.0 & 16.0 \\
& Enterococcusspp & 6 & 14.0 & 13.0 & 19.0 \\
& Listeria monocytogenes & 2 & 11.0 & 11.0 & 11.0 \\
\hline
\end{tabular}

Elaborado por: los autores. Fuente: base de datos.

En tabla $N^{\circ} 2$, la mediana del tiempo de las bacterias gramnegativas para positivización fue de 13 horas con un mínimo de 6 y un máximo de 42; para las grampositivas fue de 14, siendo el menor 7 y el mayor, 42 horas.

Obtenido los microorganismos a partir de los cultivos, se procedió a realizar el antibiograma correspondiente y el perfil de resistencia. 


\section{Tabla $N^{\circ} 3$}

Principales microorganismos gramnegativos aislados en los episodios bacteriemias y su perfil de resistencia a los antimicrobianos en el Instituto del Cáncer SOLCA. Cuenca, 2011 - 2016

\begin{tabular}{|c|c|c|c|c|c|c|c|c|c|c|c|c|c|}
\hline \multirow[b]{2}{*}{$\begin{array}{l}\text { Microorga- } \\
\text { nismo }\end{array}$} & \multicolumn{2}{|c|}{$\begin{array}{l}\text { Aislamien- } \\
\text { tos }\end{array}$} & \multicolumn{11}{|c|}{ Antibióticos } \\
\hline & 苋 & 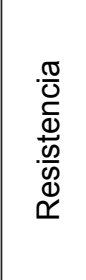 & 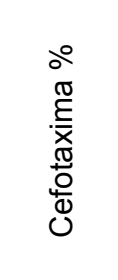 & 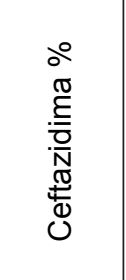 & 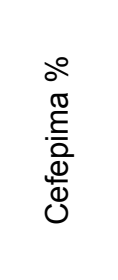 & $\begin{array}{l}\circ \\
0 \\
\frac{0}{0} \\
\overline{0} \\
\frac{0}{0} \\
\frac{0}{0} \\
.0 \\
\frac{0}{3}\end{array}$ & 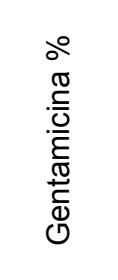 & 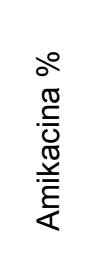 & 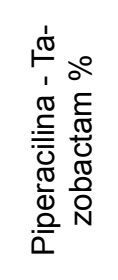 & 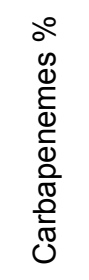 & $\sum_{i}^{\infty}$ & 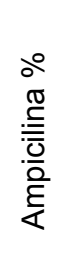 & 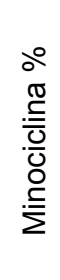 \\
\hline \multirow[t]{2}{*}{$\begin{array}{l}\text { Escherichia } \\
\text { coli }\end{array}$} & 125 & $\%$ & 29 & 29 & 31 & 35 & 17 & 6 & 23 & 0 & - & - & - \\
\hline & & $\begin{array}{l}\mathrm{n} / \\
\text { total }\end{array}$ & $36 / 125$ & $36 / 125$ & $39 / 125$ & $44 / 125$ & $21 / 125$ & $7 / 125$ & $29 / 125$ & - & - & - & - \\
\hline \multirow[t]{2}{*}{$\begin{array}{l}\text { Klebsiella } \\
\text { spp }\end{array}$} & 30 & $\%$ & 47 & 47 & 47 & 50 & 46 & 10 & 26 & 7 & - & - & - \\
\hline & & $\begin{array}{c}\mathrm{n} / \\
\text { total }\end{array}$ & $14 / 30$ & $14 / 30$ & $14 / 30$ & $14 / 28$ & $11 / 24$ & $9 / 30$ & $7 / 27$ & $2 / 30$ & - & - & - \\
\hline \multirow{2}{*}{$\begin{array}{l}\text { Pseudomo- } \\
\text { na aerugi- } \\
\text { nosa }\end{array}$} & 17 & $\%$ & - & 18 & 18 & 18 & 6 & 6 & 18 & 18 & - & - & - \\
\hline & & $\begin{array}{l}\mathrm{n} / \\
\text { total }\end{array}$ & - & $3 / 17$ & $3 / 17$ & $3 / 17$ & $1 / 17$ & $1 / 17$ & $3 / 17$ & $3 / 17$ & - & - & - \\
\hline \multirow[t]{2}{*}{$\begin{array}{l}\text { Salmonella } \\
\text { spp }\end{array}$} & 9 & $\%$ & 11 & - & - & 33 & - & - & - & - & 11 & 67 & - \\
\hline & & $\begin{array}{l}\mathrm{n} / \\
\text { total }\end{array}$ & $1 / 9$ & - & - & $3 / 9$ & - & - & - & - & $1 / 9$ & $6 / 9$ & - \\
\hline \multirow[b]{2}{*}{$\begin{array}{l}\text { Burkholde- } \\
\text { ria cepacia }\end{array}$} & 9 & $\%$ & - & 11 & - & - & - & - & - & 22 & 22 & - & 33 \\
\hline & & $\begin{array}{l}\mathrm{n} / \\
\text { total }\end{array}$ & - & $1 / 9$ & - & - & - & - & - & $2 / 9$ & $2 / 9$ & - & $3 / 9$ \\
\hline \multirow[b]{2}{*}{$\begin{array}{l}\text { Enterobac- } \\
\text { ter cloacae }\end{array}$} & 7 & $\%$ & 28 & 28 & - & - & 14 & 14 & 14 & - & - & - & - \\
\hline & & $\begin{array}{l}\mathrm{n} / \\
\text { total }\end{array}$ & $2 / 7$ & $2 / 7$ & - & - & $1 / 7$ & $1 / 7$ & $1 / 7$ & - & - & - & 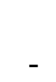 \\
\hline
\end{tabular}

Elaborado por: los autores. Fuente: base de datos.

Para cada uno de los microorganismos, en la primer fila consta el porcentaje determinado a partir de relacionar el número de aislamientos resistentes con el total muestras en las que se aisló el microorganismo señalado y que en fracción, consta en la segunda fila.

A partir del análisis de la tabla $\mathrm{N}^{\circ} 3$ en el perfil de resistencia podemos destacar: E. coli: de 125 cepas, $29 \%$ fueron $\mathrm{R}$ a cefotaxima y ceftazidima por el mecanismo de Beta-lactamasa de espectro extendido (BLEE), 31\% $\mathrm{R}$ a cefepima; no hubo re- sistencia a carbapenemes. Klebsiella. spp: de los 30 aislamientos, el $47 \%$ fueron $\mathrm{R}$ cefotaxima, ceftazidima y cefepima con el fenotipo de BLEE y 2 de esas cepas presentaron resistencia a carbapenemes por carbapenemasas. $P$. aeruginosa: en $3 / 17$ cepas se encontró $R$ a ceftazidima, cefepima, ciprofloxacina, piperacilina-tazobactam y carbapenemes; una de ellas $\mathrm{R}$ a aminoglucósidos. Salmonella spp: en $1 / 9$ se observó $R$ a cefotaxima, 1/9 R a TMS, 6/9 R a ampicilina y 3/9 $R$ a ciprofloxacina. B. cepacia: del total de 9 aislamientos, 1 fue $\mathrm{R}$ a ceftazidima, $2 \mathrm{R}$ a TMS, $2 \mathrm{R}$ a Meropenem y $3 \mathrm{R}$ a 
minociclina. Enterobacter cloacae: 2/7 aislamientos $\mathrm{R}$ a cefotaxima y ceftazidima, una de ellas con el fenotipo de BLEE, 1/7 $R$ a piperacilina-tazobactam y $1 / 7 R$ a aminoglucósidos.

\section{Tabla $N^{\circ} 4$}

Perfil de resistencia a los antimicrobianos en las principales bacterias grampositivas identificadas en los episodios bacteriemias en pacientes oncológicos del Instituto del Cáncer SOLCA. Cuenca, 2011 - 2016

\begin{tabular}{|c|c|c|c|c|c|c|c|c|c|c|c|c|c|}
\hline \multirow[b]{2}{*}{$\begin{array}{l}\text { Microorganis- } \\
\text { mo }\end{array}$} & \multicolumn{2}{|c|}{ Aislamientos } & \multicolumn{11}{|c|}{ Antibióticos } \\
\hline & Total & 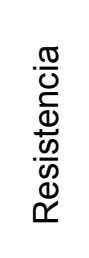 & 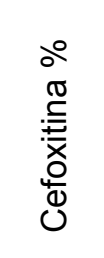 & 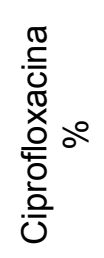 & $\sum_{i}^{\infty}$ & 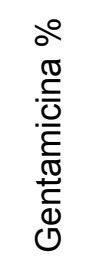 & 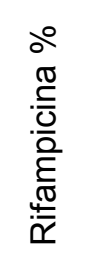 & 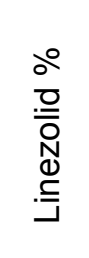 & 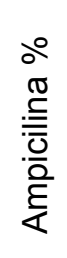 & 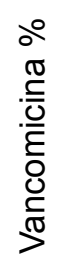 & 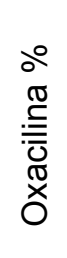 & 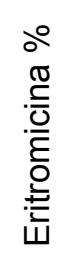 & 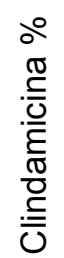 \\
\hline \multirow[b]{2}{*}{$\begin{array}{l}\text { Staphylococ- } \\
\text { cus aureus }\end{array}$} & 60 & $\%$ & 40 & 19 & 35 & 13 & 9 & 0 & - & - & - & - & - \\
\hline & & $\begin{array}{c}\mathrm{n} / \\
\text { total }\end{array}$ & $24 / 60$ & $11 / 60$ & $21 / 60$ & $9 / 60$ & $5 / 60$ & $0 / 60$ & - & - & - & - & - \\
\hline \multirow{2}{*}{$\begin{array}{l}\text { Staphylococ- } \\
\text { cus coagula- } \\
\text { sa negativa }\end{array}$} & 24 & $\%$ & 67 & 68 & 78 & 44 & 10 & 0 & - & - & - & - & - \\
\hline & & $\begin{array}{c}\mathrm{n} / \\
\text { total }\end{array}$ & $16 / 24$ & $15 / 22$ & $18 / 23$ & $8 / 18$ & $2 / 19$ & $0 / 24$ & - & - & - & - & - \\
\hline \multirow[t]{2}{*}{ Enterococos } & 6 & $\%$ & - & - & - & - & - & - & 33 & 17 & - & - & - \\
\hline & & $\begin{array}{c}\mathrm{n} / \\
\text { total }\end{array}$ & - & - & - & - & - & - & $2 / 6$ & $1 / 6$ & - & - & - \\
\hline \multirow{2}{*}{$\begin{array}{l}\text { Streptococ- } \\
\text { cus pneumo- } \\
\text { niae }\end{array}$} & 6 & $\%$ & - & - & - & - & - & - & - & - & 0 & - & - \\
\hline & & $\begin{array}{c}\mathrm{n} / \\
\text { total }\end{array}$ & - & - & - & - & - & - & - & - & $0 / 6$ & - & - \\
\hline \multirow{2}{*}{$\begin{array}{l}\text { Streptococ- } \\
\text { cus betahe- } \\
\text { molitico }\end{array}$} & 6 & $\%$ & - & - & - & - & - & - & - & - & - & 33 & 33 \\
\hline & & $\begin{array}{c}\mathrm{n} / \\
\text { total }\end{array}$ & - & - & - & - & - & - & - & - & - & $2 / 6$ & $2 / 6$ \\
\hline
\end{tabular}

Elaborado por: los autores. Fuente: base de datos.

Las resistencias a destacar de la tabla $\mathrm{N}^{\circ}$ 4: $\mathrm{S}$. aureus: el $40 \%$ fueron $\mathrm{R}$ a cefoxitina Staphylococcus aureus metilicino resistente (SAMR), no se encontró resistencia a linezolid. ECN: 16/24 fueron $\mathrm{R}$ a cefoxitina, y ninguna cepa fue resistente a linezolid. Enterococcus spp: 2/6 cepas fueron $\mathrm{R}$ a ampicilina y una de ellas $\mathrm{R}$ a vancomicina. Todas fueron aptas para sinergia con gentamicina y estreptomicina de alta carga. S.pneumoniae: los seis aislamientos fueron sensibles a oxacilina.

No fue posible documentar microbiológicamente el foco de la bacteriemia por cuanto el número de muestras de otros sitios que ingresaron al laboratorio para cultivo, fue mínimo.

\section{Discusión}

Las infecciones en los pacientes oncológicos son producidas por gérmenes que ganan acceso a sitios estériles a través de las barreras epiteliales alteradas por la quimioterapia y la inmunosupresión; la complicación de éstas puede llevar a la bacteriemia que representa una importante causa de morbilidad y mortalidad en estos pacientes [14].

La distribución de los agentes etiológicos mostró un predominio de las bacterias gramnegativas (66.8\%), mientras que los grampositivos fueron responsables del $33.2 \%$. En Francia, entre 2003 y 2010, Bousquet A, et al., se confirmó este predomi- 
nio; los porcentajes fueron del $70.8 \%$ y $18.7 \%$, respectivamente [15]. Esto contrasta con la mayoría de las publicaciones que muestran preponderancia de las bacterias grampositivas en los últimos años, en población con cáncer tanto pediátrica como adulta, donde los microorganismos más frecuentemente recuperados pasaron a ser los Staphylococcuscoagulasa negativa, S. aureus y más recientemente estreptococos del grupo viridans y enterococos, en comparación a las prevalencias observadas a inicios de los años 70 donde los gramnegativos eran responsables del $80 \%$ de las infecciones bacterianas [4,8,16-18] Al parecer, el uso de dispositivos para canalizar vías periféricas y centrales, está implicado en el cambio.

En otra serie de pacientes neutropénicos de 9 hospitales franceses y uno de Bélgica, se aislaron 1147 microorganismos de los cuales, 88 bacteriemias fueron polimicrobianas; los patógenos incluyeron E. coli en el $10.7 \%$, Klebsiella-Enterobacter-Serratia $(6.1 \%)$, otras enterobacterias $(2.2 \%)$, P. aeruginosa (4.8\%), otros BNF (4.7\%), Staphylococcus coagulasa negativa $(40.8 \%)$, S. aureus $(9.9 \%)$, estreptococos $(5.4 \%)$, enterococos $(2.2 \%)$, anaerobios (3.4\%), otras bacterias con el 6.9\% [19]. Los resultados se relación con lo ya afirmado, siendo conveniente tomar en cuenta las condiciones higiénico sanitarias y el nivel de educación de la población.

Es importante resaltar que en nuestra serie se detectaron 9 bacteriemias por Salmonella spp.; las enfermedades inmunosupresoras como leucemias y linfomas en las que aumenta la frecuencia de metástasis focales, son factores de riesgo que favorecen el desarrollo de este microorganismo [20,21]. Del mismo modo, aislamientos de Aeromonas spp. en sangre, se describen en la literatura en pacientes con enfermedades malignas como las neoplasias o enfermedades hepáticas [22].

Las infecciones polimicrobianas, a menudo entre bacterias anaerobias y aerobias, ocurren en el 5 a $20 \%$ de los casos [23]. En nuestra población, cuatro episodios correspondieron a infecciones polimicrobianas lo que representó un $1.3 \%$. La diferencia quizá tenga que ver con la gravedad del paciente.

En cuanto a las bacteriemias por grampositivos, S. aureus se aisló en el $17.9 \%$ de los episodios. Este hallazgo confirma la tendencia observada en la literatura médica donde $\mathrm{S}$. aureus fue la causa del $11-33 \%$ de las bacteriemias nosocomiales según un estudio realizado en Bogotá [24]. Los Staphylococcus coagulasa negativa le siguieron en frecuencia y en menor proporción; también se aislaron S. pneumoniae, Streptococcus beta hemolítico del grupo C (SBGC) y enterococos. El $1.8 \%$ de las bacteriemias por grampositivos correspondió a SBGC. Diversos estudios demuestran que más del $80 \%$ de las bacteriemias por Streptococcus dysgalactiae sub $\mathrm{sp}$. equisimilis (SBGC o G) se relacionan con la presencia de varias comorbilidades entre las cuales las neoplasias son las más frecuentes [25].

En la literatura se observa que la mayoría de las bacteriemias se detectan dentro de las primeras 48 horas de incubación, cuando se emplean métodos automatizados de monitoreo continuo de hemocultivos [7]; En el estudio que se presenta, el $97 \%$ de los microorganismos se desarrollaron dentro de las 24 horas de incubación y el 3\% restante antes de las 48 horas, con una mediana de 13 horas para bacterias gramnegativas y de 14 horas para grampositivas.

Con respecto a la resistencia a los antimicrobianos con el fenotipo de BLEE fue encontrado un $29 \%$ de E. coli y 47\% de K. pneumoniae: en el año 2007 en EE.UU, la prevalencia de BLEE fue del 5 al $10 \%$ ( $7.5 \%$ en E. coli y $12,3 \%$ en K. pneumoniae) [26], mientras que en Europa, las BLEE fueron el $22.6 \%$ de K. pneumoniae y $5.3 \%$ de E. coli [27]; sin embargo, ya en el año 2003 la Sociedad Española de Enfermedades Infecciosas y Microbiología Clínica (SEIMC) publicó que las BLEE provenientes de 40 hospitales españoles correspondían al $64.9 \%$ en E. coli y a $86.4 \%$ en K. pneumoniae [28]. En una revisión de la literatura sobre bacteriemias en pacientes con cáncer en 18 países de Europa, las enterobacterias productoras de BLEE en adultos representaron el $34 \%$ ( $42 \%$ para E. coli) y $18 \%$ en niños [29].

Respecto a Salmonella, en los últimos años aparecieron resistencias a diferentes antibióticos con un creciente número de informes relacionados con la disminución de sensibilidad a fluorquinolonas que es alarmante [30]. En nuestro estudio, 6 de 9 cepas fueron $R$ a ampicilina y 3 cepas fueron $R$ a ciprofloxacina. 
En S. aureus, el $40 \%$ fueron MR, lo que concuerda con el $30-50 \%$ que describe la literatura, la que señala que existen grandes variaciones entre un hospital y otro e incluso entre diferentes países; en ECN la R a meticilina fue casi del $70 \%$, coincidiendo con lo publicado en otras series [4].

En años recientes en Egipto, bacterias con resistencia múltiple a los antibióticos conocidas como ESKAPE: (Enterobacter spp., SAMR, K. pneumoniae, A. baumannii, P. aeruginosa y enterococos) han sido identificadas como responsables de bacteriemias en pacientes con cáncer, con prevalencias de dichos microorganismos que varían entre un $12 \%, 23 \%, 37 \%, 10 \%$ y $9 \%$ respectivamente [31].

\section{CONCLUSIONES:}

Los agentes etiológicos causantes de los episodios estudiados son predominantemente los bacilos gramnegativos, ocupando el primer lugar E. coli. seguida de K. pneumoniae, y P. aeruginosa.

Se encontró que el fenotipo BLEE estaba presente en un $29 \%$ en E. coli y $47 \%$ en K. pneumoniae y dos cepas de K. fueron resistentes a los carbapanemicos.

En cuanto a los cocosgrampositivos $\mathrm{S}$. aureus el $40 \%$ fueron $R$ a cefoxitina (SAMR), 35\% R a TMS, no se encontró resistencia a linezolid.

Un mayor número de episodios de bacteriemias se dieron en los pacientes con tumores hematológi$\cos (57,5 \%)$ esto puede estar relacionado con la duración de la neutropenia que esta entre 14 y 21 días lo que implica mayor riesgo a infecciones.

\section{INFORMACIÓN DE LOS AUTORES}

- Mora Abad Johanna Magdalena. Licenciada en Laboratorio Clínico Especialista en Microbiología Clínica. SOLCA-Cuenca

ORCID: https://orcid.org/0000-0001-6692-5557

- Procopio Adriana Nora. Bioquímica. especialista en Microbiología Clínica. Hospital de Niños Dr. Ricardo Gutiérrez. Buenos Aires. Argentina. ORCID: https://orcid.org/0000-0002-2176-5518

- Hurtado Bustamante Pablo Francisco. Infectólogo Clínico. SOLCA-Cuenca ORCID: https://orcid.org/0000-0001-5769-3926
- Alvarado Corral Raul Francisco. Cirujano Oncólogo. SOLCA - Cuenca / Hospital Santa Inés. ORCID: https://orcid.org/0000-0003-0095-259X

- Martínez Reyes Fray Cleiton. Doctor en Medicina y Cirugia. Magister en investigación de la salud. SOLCA - Cuenca / Universidad del Azuay. ORCID: https://orcid.org/0000-0003-3363-2402

\section{CONTRIBUCIÓN DE LOS AUTORES}

MA: participación en la idea del trabajo y todo el proceso de investigación

PA: revisión de los diferentes productos del proceso de investigación

HB: revisión del informe final

AC: revisión del informe final

MR: participación en todo el proceso de investigación

\section{CONFLICTO DE INTERESES}

Los autores declaran no tener conflicto de intereses en la presente investigación.

\section{FUENTES DE FINANCIAMIENTO}

Autofinanciamiento

\section{REFERENCIAS BIBLIOGRÁFICAS}

1. Macedo M, Algorta G, Vola M, Pardo L. Temas de Bacteriología y Virología Medica. Bacteriemias y sepsis. Endocarditis. 2da edición, Uruguay, Oficina del libro FEFMUR. 2006 p. 197-211.

2. Artico MJ, Rocchi M, Gasparotto A, Ocaña Carrizo V, Navarro $M$, Mollo $V$, et al. Community-acquired bacteremia in adult patients attending the emergency service of a teaching hospital. Rev Argent Microbiol. 2012;44(1):10-5.

3. Rubio I, Ferreiro J, Pérez T. Infecciones en el paciente oncológico. Neutropenia febril. Gac Médica Bilbao.2004;101(2):53-8. 
4. Cheguirián ML, Carvajal LR, Ledesma EM, Enrico MC, Reale AL, Culasso $\mathrm{C}$, et al. Prevalencia de microorganismos causantes de bacteriemias y fungemias en pacientes oncológicos pediátricos. Patrones de sensibilidad a los antimicrobianos. Rev Argent Microbiol. 2008; 40(2):111-15.

5. García-Gómez M, Guío L, Hernández JL, Vilar B, Pijoán JI, Montejo JM. Bacteriemias por enterobacterias productoras de betalactamasas (BLEE, AmpC y carbapenemasas) asociación con los cuidados sanitarios y los pacientes oncológicos. Rev Esp Quimioter. 2015;28(5):256-62.

6. Nielsen SL. The incidence and prognosis of patients with bacteremia. Dan Med J. 2015;62(7).

7. Soloaga R. Criterios de interpretación de hemocultivos análisis crítico de diversas pautas. Bioanalisis 2018;36:14-26. Disponible en: http://www.revistabioanalisis.com/arxius/ notas/nota3.pdf

8. Fortún J, Sanz MÁ, Madero L, López J, de la Torre J, Jarque I, et al. Update on bacteraemia in oncology and hematology. Enferm Infecc Microbio IClin. 2011; 29(4):48-53.

9. Romero RM, Acosta DDM, Ortega AB. Prevalencia y resistencia antimicrobiana de microorganismos aislados en el Centro Oncológico Estatal del ISSEMYM. Rev Latinoam Patol Clin Med Lab. 2013; 60:244251.

10. Cortez D, Rodríguez N, Benadof D, Zamorano A, Tordecilla J. Bacteriemia en pacientes oncológicos. Experiencia en un hospital pediátrico. Rev Chil Infect. 2012;29(2):16468.

11. LaluezaA. Importancia actual de la bacteriemia por" staphylococcus aureus" en un hospital universitario. Universidad Complutense de Madrid; 2008.

12. Hinojosa-AndíaLJ, Carpio-JayoD.Bacteriemia asociada a neutropenia febril en pacientes hemato-oncológicos, su espectro bacteriano y patrón de susceptibilidad antibiótica. Rev Medica Hered. 2014;25(1):22-29.

13. Cisneros-Herreros JM, Cobo-Reinoso J, Pujol-Rojo M, Rodríguez-Baño J, SalavertLletí M. Guía para el diagnóstico y tratamiento del paciente con bacteriemia. Guías de la Sociedad Española de Enfermedades Infecciosas y Microbiología Clínica (SEIMC). Enferm Infecc Microbiol Clin. 2007;25(2):11130.

14. Galloway-Peña J, Brumlow C, Shelburne S. Impact of the Microbiota on Bacterial Infections during Cancer Treatment. Trends Microbiol. 2017;25(12):992-1004.

15. Bousquet A, Malfuson J-V, Sanmartin N, Konopacki J, MacNab C, Souleau B, et al. An 8-year survey of strains identified in blood cultures in a clinical haematology unit. Clin Microbiol Infect. 2014;20(1):07-012.

16. Mebis J, Jansens H, Minalu G, Molenberghs G, Schroyens W, Gadisseur A, et al. Longterm epidemiology of bacterial susceptibility profiles in adults suffering from febrile neutropenia with hematologic malignancy after antibiotic change. Infect Drug Resist. 28 de julio de 2010;3:53-61.

17. Miedema KGE, Winter RHLJ, Ammann RA, Droz S, Spanjaard L, de Bont ESJM, et al. Bacteria causing bacteremia in pediatric cancer patients presenting with febrile neutropenia--species distribution and susceptibility patterns. Support Care in Cancer . 2013;21(9):2417-26. Disponible en: https://doi.org/10.1007/s00520-013-1797-4 
18. Aust $\mathrm{C}$, Tolfvenstam $\mathrm{T}$, Broliden $\mathrm{K}$, Ljungman P, Kalin M, Giske CG, et al. Bacteremia in Swedish hematological patients with febrile neutropenia: Bacterial spectrum and antimicrobial resistance patterns. Scand J Infect Dis. 1 de abril de 2013;45(4):285-91.

19. Dumford DM, Skalweit M. Antibiotic-Resistant Infections and Treatment Challenges in the Immunocompromised Host. Infect Dis Clin North Am. 2016;30(2):465-89.

20. Frias Salcedo JA. Bacteriemia por salmonela no tifoídica en pacientes inmunocomprometidos. Enf Inf Microbiol. 2009;29(4):145-49.

21. Pila S. Bacteriemia por Salmonella. Clínica Medica; Santa Fé;2015 p.1-7.

22. Soloaga R, Abiega C, Vilaró M. Hemocultivos, Endocarditis, Catéteres y Sepsis. Herramientas para optimizar el diagnóstico microbiológico. Córdoba Argentina: Editorial Brujas; 2013.

23. Cohen J, Powderly WG, Opal SM, Calandra T, Clumeck N, Day J, et al. Infectious diseases. Infections in the cancer patient 4th edition, USA, ELSEVIER; 2017(1):723-38.

24. Cuervo SI, Cortés JA, Sánchez R, Rodríguez JY, Silva E, Tibavizco D, et al. Risk factors for mortality caused by Staphylococcus aureus bacteremia in cancerpatients. Enferm Infecc Microbiol Clin. 2010;28(6):349-54.

25. Di Bella JHD, Monterisi A, Navarro $M$, Romero $\mathrm{V}$, Novillo $\mathrm{F}$, Manassero $\mathrm{C}$, et al. Bacteriemias por Streptococcus dysgalactiae subsp. equisimilis en adultos:análisis de 36 episodios. Experiencia Médica. 2016; 34(1): 05-09.

26. Turner PJ. Extended-Spectrum b-Lactamases. Clin Infect Dis. 2005 15(4):273-75.

27. Pfaller MA, Jones RN, Doern GV, Kugler K. Bacterial Pathogens Isolated from Patients with Bloodstream Infection: Frequencies of Occurrence and Antimicrobial Susceptibility Patterns from the SENTRY Antimicrobial Surveillance Program (United States and Canada, 1997). Antimicrob Agents Chemother. 1998; 42(7):1762-770.

28. Casellas JM. Informe del Comité de Resistencia a antibacterianos de la API período 2003-2004. Rev Panam Infectol. 2004;6:28-30.

29. Mikulska M, Viscoli C, Orasch C, Livermore DM, Averbuch D, Cordonnier C, et al. Aetiology and resistance in bacteraemias among adult and paediatric haematology and cancer patients. J Infect. abril de 2014;68(4):321-31.

30. Informe sobre la resistencia de Salmonella spp. en las Américas. Rev Panam Pública/ Pan Am J Public Health. 2006;19(2).

31. El-Mahallawy HA, Hassan SS, El-Wakil M, Moneer MM. Bacteremia dueto ESKAPE pathogens: Anemerging problem in cancer patients. J Egypt Natl Cancer Inst. septiembre de 2016;28(3):157-62. 